Le congrès "Santé des médecins - de leurs études à la retraite» axé sur la chronologie de la vie professionnelle a passé en revue les différents aspects de la santé des médecins. Un point de vue partagé par de nombreux participants s'est cependant imposé comme le fil rouge de cette journée qui a mis deux souhaits en lumière: le respect des valeurs cardinales de la profession de médecin et les possibilités d'interaction sociale contre la tendance à l'isolement né de la pression liée au rendement et à la sélection, et ce des études jusqu'en fin de carrière. S'agissant de l'équilibre entre la vie professionnelle et la vie privée, des solutions développées en concertation avec les médecins doivent être encouragées.

Dr med. Carlos Quinto, membre du Comité central, responsable du département Santé publique, professions de la santé et produits thérapeutiques

\title{
La «Santé pour tous», donc aussi pour les médecins
}

\section{Barbara Weil}

Cheffe de la division Promotion de la santé et prévention

L'OMS au niveau mondial aussi bien que les instances européennes rappellent régulièrement que la santé fait partie de notre patrimoine, qu'elle est un droit humain. C'est donc bien évidemment aussi le cas pour les médecins. Cette question a été au cœur du symposium du 7 avril dernier sur la "Santé des médecins - de leurs études à la retraite». Les étudiants en médecine se rendent très vite compte que leur santé sera essentielle pour poursuivre sans encombre sur la voie de leur vocation. Sensibilisation à sa santé individuelle, transmission des compétences personnelles et le contact avec ses pairs ne sont là que quelques exemples d'idées qu'il serait pertinent d'approfondir. En d'autres termes, il faut mettre l'accent sur le groupe et l'entraînement aux compétences sociales, et s'éloigner de l'isolement, de la course aux objectifs d'apprentissage pour s'approcher d'une "œuvre» commune. Le succès de la mise en application de mesures de santé publique dépend souvent de l'interaction des groupes cibles lors de l'élaboration de projets et de programmes; pourquoi ne pas faire la même chose lorsqu'il s'agit de la santé des médecins?

\section{Se soigner seul n'est souvent pas la solution:} un médecin a aussi le droit d'être un patient.

Travail, formation, temps libre et famille: la coupe est souvent bien trop petite pour qu'elle ne déborde pas. Ce n'est pas seulement une question de génération ou de genre comme l'ont clairement démontré les participants, de tous âges, du symposium. Pourquoi faut-il encore aujourd'hui faire preuve de courage pour s'adresser à son supérieur et lui demander une diminution de son taux d'activité de $100 \%$ à 90\%? Le partage des postes de travail, un sujet d'actualité, semble ne pas être aussi aberrant que ça; plusieurs exemples montrent que cela peut aussi très bien fonctionner en Suisse.
Chacun doit être à l'écoute et prendre soin de sa propre personne, surtout avant qu'une situation de crise ne s'installe. Se soigner seul n'est souvent pas la solution: un médecin a aussi le droit d'être un patient. Une assistante médicale pourrait-elle l'aider à ne pas dire non à la consultation chez un collègue? Est-ce que je me sens capable de traiter un confrère ou une consœur? A quels points et à quelles conditions cadres faut-il faire attention pour qu'un traitement entre pairs se passe bien?

\section{La santé individuelle est essentielle pour} poursuivre sur la voie de sa vocation.

Les institutions du domaine de la santé n'ont bien souvent que des possibilités limitées de mettre en œuvre un projet de «skill and grade mix» (un savant équilibre entre les compétences de métier [skill] et les niveaux de formation [grade] au sein des équipes de soins). Il est aussi évident qu'il importe de se concentrer davantage sur la prévention structurelle (groupe) sans perdre des yeux la prévention comportementale (individu). Enfin, l'excès de bureaucratie dessert autant le patient que le médecin et ne fait que générer un cimetière de données, dont personne ne peut tirer aucune plusvalue. Mais des idées séduisantes existent, elles nous viennent de Nouvelle-Zélande par exemple où un cadre de l'administration est chargé de veiller à ce que l'objectif «No paperwork for doctors» puisse être respecté; mais aussi d'Allemagne, où le métier de documentaliste hospitalier s'est largement établi. Cette profession s'adresse à un personnel spécialisé qui a suivi une formation (master) dans une haute école.

Parfois, il suffit de forcer un regard curieux au-delà de son jardin pour générer de nouvelles idées. Allons-y! 